\title{
Changes in Heterosis of Maize (Zea mays L.) Varietal Cross Hybrids after Four Cycles of Reciprocal Recurrent Selection
}

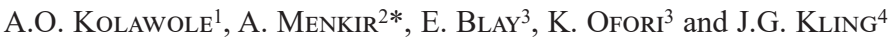 \\ ${ }^{1}$ Ladoke Akintola University of Technology, Ogbomoso, Nigeria \\ ${ }^{2}$ International Institute of Tropical Agriculture (IITA), Oyo Road, PMB 5320, Ibadan, Nigeria \\ ${ }^{3}$ West Africa Centre for Crop Improvement (WACCI), University of Ghana, Legon \\ ${ }^{4}$ Department of Crop and Soil Science, College of Agricultural Sciences, Oregon State University, \\ Corvallis, OR 97331
}

(Received 17, July 2018; Accepted 16 October 2018)

Two improved tropical maize composites, TZL COMP3 and TZL COMP4; representing complementary heterotic pools have been subjected to four cycles of reciprocal recurrent selection (RRS) for two decades to enhance varietal cross performance. The objectives of this study were to evaluate the effect of selection on genetic gain in heterosis for grain yield and other agronomic traits of these composites. Ten parental populations representing the $\mathrm{C}_{0}$ to $\mathrm{C}_{4}$ of each composite and their crosses plus a varietal check were evaluated in a trial at eight environments in Nigeria. Grain yield of the varietal crosses increased with selection by $3.1 \%$ cycle $^{-1}$. Mean grain yields of the $\mathrm{C}_{4} \times \mathrm{C}_{4}$ varietal cross exceeded that of a popular improved reference variety by $23 \%$. Selection also reduced anthesis-silking interval, improved ear characteristics, phenotypic appeal and resistance to foliar diseases. Mid-parent heterosis (MPH) increased from $4 \%$ at $\mathrm{C}_{0} \times \mathrm{C}_{0}$ to $24 \%$ at $\mathrm{C}_{4} \times \mathrm{C}_{4}$. The average rate of genetic gain in heterosis for grain yield in population crosses was $3.1 \%$ possibly because of presence of non-additive gene effects. The results of our study present the potential usefulness of the advanced selection cycle as sources of diverse inbred lines with improved combining ability as well as improved varietal crosses that can be multiplied and deployed in areas with limited market access.

Keywords: genetic gain, heterosis, composites, varietal crosses

\section{Introduction}

Maize (Zea mays L.) is a major staple food and primary source of daily calorie for humans in several countries in sub-Saharan Africa (Olaniyan 2015). Increasing maize productivity per unit area will lead to more food, reduced food prices for the urban and rural poor and extra produce for sale. The development and commercialization of maize varieties and hybrids with high yield potential is therefore important for increased productivity in the diverse production environments (Menkir et al. 2006). This can be achieved through improvements in breeding efficiency to accelerate the rate of genetic gains. 
In hybrid maize breeding programs, interpopulation improvement methods are used to exploit heterosis in grain yield and other traits (Ordas et al. 2012). Comstock et al. (1949) describes reciprocal recurrent selection (RRS) as one of the cyclical breeding methods that exploits additive and nonadditive genetic effects in the population cross involving two genetically diverse populations that serve as reciprocal tester for each other. Such complementary source population can be used by breeders after improvement for grain yield and other traits (Lamkey and Hallauer 1987). Reciprocal recurrent selection were thus designated to gradually increase the frequency of favorable alleles in complementary populations that allow the development of divergent populations and inbred lines from advanced selection cycles to maximize heterosis (Hallauer and Miranda 1995; Betrán and Hallauer 1996). Breeders at the International Institute of Tropical Agriculture (IITA) have then created two late maturing tropical maize composites, designated as TZL COMP3 and TZL COMP4, for a long-term RRS program to maximize expression of heterosis in hybrids. These composites have been subjected to four cycles of RRS to boost agronomic performance of hybrids formed from inbred lines and varieties developed from advanced selection cycles (Falconer and Mackay 1996).

Several studies have shown that the level of heterosis expressed in population crosses for grain yield and other desirable agronomic traits has been enhanced through RRS (Menz and Hallauer 1997; Menz et al. 1999; Carena and Hallauer 2001). Such improvement in heterosis through RRS allow the development of superior hybrids (Reis et al. 2013) and population crosses that can be used as commercial varieties (Carena 2005; Carena and Wicks III 2006). These hybrids may exhibit heterosis as a consequence of genetic divergence and partial to complete dominance of genes controlling grain yield and other traits (Hallauer and Miranda 1995). When population crosses are used as commercial hybrids that can be profitable alternatives to single-cross hybrids (Carena 2005; Souza Jr et al. 2010; Jenweerawat et al. 2010), yield depression which occurs with the recycling of seed is lower in the former than in the latter (Paliwal 2000). Likewise, the ease of seed production and low seed price of improved varietal crosses may be of interest to small holder farmers' (Mosisa et al. 2009) in remote rural areas with limited market access. Several studies involving populations improved through RRS documented increases in heterosis for grain yield varying from $2.5 \%$ at $\mathrm{C}_{0}$ to $42.5 \%$ at the advanced selection cycle (Souza Jr and Pinto 2000; Santos et al. 2007; Romay et al. 2011; Reis et al. 2013).

Although TZL COMP3 and TZL COMP4 had undergone four cycles of RRS, however, the gain in heterosis in these composites has not been assessed to determine the development of both non-conventional and conventional hybrids attractive to indigenous seed companies depending on their technical skills to produce and market hybrids. This study was, therefore, conducted to evaluate the effect of selection on genetic gain in heterosis for grain yield and other agronomic traits in varietal crosses of the original and advanced selection cycles of the two tropical maize composites. 


\section{Materials and Methods}

\section{Genetic materials}

Details about the components and the steps followed to constitute the two composite TZL COMP $3 \mathrm{C}_{0}$ and TZL COMP4 $\mathrm{C}_{0}$ have been described in detail in the Maize Improvement Program report (MIP, 1996). Also, the selection procedures used to improve the first $\left(\mathrm{C}_{1}\right)$ to the fourth $\left(C_{4}\right)$ selection cycles has been described in detail by Menkir et al. (2015). In 2013 , the original $\left(\mathrm{C}_{0}\right)$ and advanced $\left(\mathrm{C}_{1}, \mathrm{C}_{2}, \mathrm{C}_{3}\right.$ and $\left.\mathrm{C}_{4}\right)$ selection cycles of TZL COMP3 and TZL COMP4 were increased using 1000 plant-to-plant crosses in each selection cycle.

\section{Trial evaluation}

The two original $\left(\mathrm{C}_{0}\right)$ and eight advanced selection cycles $\left(\mathrm{C}_{1}, \mathrm{C}_{2}, \mathrm{C}_{3}\right.$ and $\left.\mathrm{C}_{4}\right)$ of the two composites and their 5 varietal crosses plus a popular improved reference variety (IWD C2 SYN) were evaluated in 2014 and 2015 (Table S1*) at Ikenne (forest, $3^{\circ} 42^{\prime} \mathrm{E}, 6^{\circ} 54^{\prime} \mathrm{N}$, altitude $30 \mathrm{~m}$ ), Saminaka (savanna mid-altitude transitional zone, $8^{\circ} 39^{\prime} \mathrm{E}, 10^{\circ} 34^{\prime} \mathrm{N}$, altitude $760 \mathrm{~m}$ ), Zaria (Northern Guinea savanna, $7^{\circ} 21^{\prime} \mathrm{E}, 1^{\circ} 7^{\prime} \mathrm{N}$, altitude $640 \mathrm{~m}$ )) and Kadawa (Sudan savanna, $8^{\circ} 19^{\prime} \mathrm{E}, 12^{\circ} 2^{\prime} \mathrm{N}$, altitude $520 \mathrm{~m}$ ) in Nigeria. The trial was arranged in a $4 \times 4$ lattice design with three replicates. At each location, a genotype was planted in a two-row plot, $5 \mathrm{~m}$ long with $0.75 \mathrm{~m}$ spacing between rows and $0.50 \mathrm{~m}$ spacing between hills. Three maize seeds were sown per hill and later thinned to two plants per hill at two weeks after sowing ( 2 WAS) in order to leave 22 plants in each row. A compound fertilizer was applied at the rates of $60 \mathrm{~kg} \mathrm{~N} \mathrm{ha}^{-1}, 60 \mathrm{~kg} \mathrm{P} \mathrm{ha}^{-1}$ and $60 \mathrm{~kg} \mathrm{~K} \mathrm{ha}^{-1}$ at the time of sowing. An additional $60 \mathrm{~kg} \mathrm{ha}^{-1} \mathrm{~N}$ was applied in the form of urea for top dressing four weeks later. In each trial, gramoxone and primextra were applied as pre-emergence herbicides, followed by manual weeding to keep the trials weed-free.

\section{Collection of agronomic data}

In each plot, days to anthesis and silking were recorded as the number of days from planting to when $50 \%$ of the plants shed pollen and had emerged silks, respectively. The anthesis-silking interval (ASI) was computed as the interval in days between anthesis and silking. Plant and ear heights were recorded in $\mathrm{cm}$ as the average measurements on 20 competitive plants per plot and were measured after anthesis from the base of the plant to the height of the first tassel branch and the node bearing upper ear, respectively. Husk cover was rated on a scale of 1 to 5 , where $1=$ husks tightly arranged and extended beyond the ear tip and $5=$ ear tips exposed. Plant aspect was rated on a scale of 1 to 5 based on overall phenotypic appeal, where $1=$ excellent phenotypic appeal and $5=$ poor phenotypic appeal. Ear aspect was scored on a 1 to 5 scale, where $1=$ clean, uniform, large and well-filled ears and $5=$ rotten, variable, and small ears. Ears per plant were recorded as the number of ears with fully developed grain divided by the number of harvested plants.

*Further details about the Electronic Supplementary Material (ESM) can be found at the end of the article. 
Symptom severity of southern corn leaf rust caused by Puccinia polysora, Curvularia leaf spot caused by Curvularia lunata and Maize streak virus transmitted by Cicadulina leaf hoppers were scored at Ikenne, which is a hot spot location for disease screening, in 2014 and 2015 on a scale of 1 to 5 , where $1=$ slight leaf infection and $5=$ severe leaf infection. Southern corn leaf blight caused by Bipolaris maydis was also scored at Ikenne just for one season, as symptoms were not observed in the second season. All ears harvested from each plot were shelled to determine percentage moisture and grain yield adjusted to $15 \%$ moisture content was computed.

\section{Statistical analysis}

A combined analysis of variance (ANOVA) for each trait was computed using PROC GLM in SAS (SAS Institute 2010), for all location-year combination which were considered environments. In this analysis, parental populations and varietal crosses were considered as fixed effects whereas replications, blocks and environments were considered as random effects. Least-squares means for varietal crosses and their parent populations were calculated across environments. The levels of significance between means were determined using Fishers protected least significant difference test (LSD) at $(P<0.05)$. Direct response to selection was calculated by regressing means of each trait of the varietal crosses or parental population within each composite as a dependent variable on the number of selection cycles (0-4) as independent variable (Eberhart 1964). The linear regression coefficient ( $b$-value) provided an estimate of the gain per cycle, which was divided by the intercept and multiplied by 100 to obtain percent response per cycle of selection (Hallauer and Miranda 1995). Mid-parent heterosis (MPH) was calculated for each trait using the formula of Falconer and Mackay (1996). Tests for significance in heterosis were calculated for each trait using a t-test (Singh and Chaudhary 2006). Genetic gains in heterosis for each trait were calculated by regressing MPH of the varietal crosses on the number of selection cycles.

\section{Results}

\section{Agronomic performance of parental populations and varietal crosses}

In the combined analyses of variance, environment significantly $(P<0.001)$ affected grain yield and other agronomic traits, except southern corn leaf rust and southern corn leaf blight (Table S2). The variance among genotypes was significant for all traits, except for ASI, ears per plant and foliar diseases. Partitioning of genotypes sum of squares into parental populations and varietal crosses also showed significant $(P<0.001)$ differences in grain yield and other agronomic traits. The genotypes $\times$ environment interaction mean squares were significant $(P<0.001)$ for grain yield, husk cover and ear aspect but not for other traits. The parental population $\times$ environment interaction was significant $(P<0.001)$ for grain yield and ear aspect whereas the varietal cross $\times$ environment interaction was significant for husk cover, ear aspect and maize streak virus disease (Table S2). 


\begin{tabular}{|c|c|c|c|c|c|c|c|c|c|c|c|c|c|c|c|c|c|c|c|c|c|}
\hline & 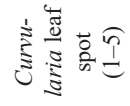 & & $\stackrel{\circ}{i}$ & 9 & $\stackrel{i}{i}$ & $\stackrel{i}{i}$ & $\stackrel{9}{-}$ & $\stackrel{\infty}{\substack{i \\
\uparrow}}$ & & $\vec{i}$ & $\overrightarrow{\mathrm{i}}$ & 9 & $\vec{i}$ & $\vec{i}$ & $\stackrel{2}{0}$ & $\stackrel{i}{i}$ & ?3. & & $\stackrel{\circ}{i}$ & & $\stackrel{\circ}{i}$ \\
\hline & 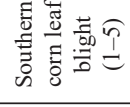 & & 9 & $\stackrel{0}{-}$ & 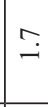 & $\tilde{i}$ & $\vec{i}$ & $\begin{array}{l} \pm \\
0 \\
0\end{array}$ & & 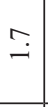 & $\stackrel{9}{-}$ & $\overrightarrow{\mathrm{i}}$ & $\stackrel{9}{-}$ & $\stackrel{I}{-}$ & $\begin{array}{l}5 \\
0 \\
\dot{9}\end{array}$ & 9 & $\ddot{0}$ & & $\stackrel{n}{-}$ & & $\cong$ \\
\hline & 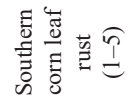 & & $\hat{i}$ & $\stackrel{n}{i}$ & $\stackrel{+}{i}$ & $\stackrel{+}{i}$ & $\stackrel{+}{i}$ & ì & & $\stackrel{n}{i}$ & $\begin{array}{l}0 \\
i\end{array}$ & $\stackrel{b}{i}$ & $\vec{i}$ & $\vec{i}$ & $\begin{array}{l}\tilde{n} \\
+ \\
+\end{array}$ & $\stackrel{+}{i}$ & ? & & $\hat{i}$ & & $\hat{i}$ \\
\hline & 总总总号号 & & 9 & $\stackrel{\sim}{i}$ & 9 & $\stackrel{9}{-}$ & $\stackrel{\infty}{-}$ & $\frac{a}{i}$ & & 9 & $\stackrel{\infty}{-}$ & तె & $\stackrel{\infty}{-}$ & $\stackrel{\infty}{-}$ & $\stackrel{\infty}{\stackrel{\infty}{+}}$ & $\stackrel{9}{-}$ & $\stackrel{3}{0}$ & & $\stackrel{\infty}{-}$ & & 9 \\
\hline & 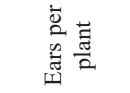 & & $\hat{o}$ & $\hat{o}$ & $\stackrel{\circ}{-}$ & $\hat{o}$ & $\dot{0}$ & กั. & & $\grave{o}$ & $\grave{o}$ & $\stackrel{\circ}{-}$ & $\grave{o}$ & $\stackrel{\circ}{-}$ & กิ & ò & $\ddot{0}$ & & oे & & $\stackrel{-}{-}$ \\
\hline & 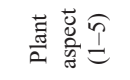 & & $\hat{i}$ & $\hat{i}$ & $\bar{i}$ & $\vec{i}$ & $\stackrel{o}{i}$ & $\frac{t}{i}$ & & $\begin{array}{l}\stackrel{\bullet}{i} \\
i\end{array}$ & $\hat{i}$ & $\tilde{n}$ & $\tilde{n}$ & $\underset{i}{0}$ & 守 & $\stackrel{0}{i}$ & $\dddot{0}$ & & $\stackrel{\mathrm{i}}{\mathrm{s}}$ & & $\stackrel{n}{i}$ \\
\hline & 矛芯 & & $\stackrel{\infty}{i}$ & $\vec{m}$ & $\stackrel{0}{\dot{r}}$ & 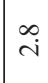 & $\hat{i}$ & $\overrightarrow{\tilde{o}}$ & & $\stackrel{\circ}{\dot{r}}$ & $\hat{\mathrm{i}}$ & $\bar{i}$ & $\bar{i}$ & $\hat{i}$ & $\stackrel{*}{\vec{i}}$ & $\stackrel{\infty}{i}$ & ڤึ & & 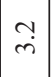 & & $\begin{array}{l}0 \\
i\end{array} \mid$ \\
\hline 坣 & 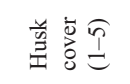 & & $\stackrel{n}{i}$ & $\stackrel{i}{i}$ & $\stackrel{+}{i}$ & $\begin{array}{l}0 \\
i\end{array}$ & $\stackrel{+}{i}$ & $\frac{n}{9}$ & & $\stackrel{n}{i}$ & $\stackrel{\tilde{i}}{\mathrm{i}}$ & $\stackrel{m}{i}$ & $\stackrel{m}{i}$ & $\stackrel{\sim}{i}$ & $\begin{array}{l}\tilde{b} \\
0 \\
0\end{array}$ & $\stackrel{+}{i}$ & ֶֻ. & & $\begin{array}{l}0 \\
i \\
i\end{array} \mid$ & & $\vec{t}$ \\
\hline & 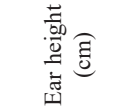 & & 苞 & $\stackrel{m}{=}$ & 㲠 & $\begin{array}{l}+ \\
\infty \\
\infty \\
0\end{array}$ & $\stackrel{\circ}{\mathscr{0}}$ & in & & $\vec{\Xi}$ & $\underset{\sigma}{\Xi}$ & 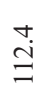 & $\begin{array}{l}0 \\
\stackrel{0}{日}\end{array}$ & $\underset{\sigma}{\ddots}$ & ָุ & $\begin{array}{l}0 \\
i \\
ٍ\end{array}$ & $\stackrel{r}{r}$ & & 苞 & & $\stackrel{?}{\stackrel{?}{\Xi}}$ \\
\hline & 䓌营氞 & & $\begin{array}{l}n \\
\text { ñ } \\
\text { ñ }\end{array}$ & $\stackrel{\vec{\theta}}{\vec{n}}$ & $\overrightarrow{\bar{\lambda}}$ & m̊̊ & 离 & i & & $\begin{array}{l}\infty \\
\underset{\sim}{~}\end{array}$ & 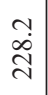 & $\frac{\infty}{\grave{a}}$ & 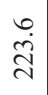 & 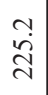 & $\ddot{0}$ & $\stackrel{+}{\infty}$ & $\stackrel{r}{i}$ & & $\begin{array}{l}\stackrel{+}{\dot{0}} \\
\stackrel{\sim}{\sim}\end{array}$ & & $\begin{array}{l}0 \\
\infty \\
\stackrel{\Delta}{v}\end{array}$ \\
\hline & 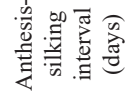 & & $\sim$ & $N$ & 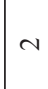 & 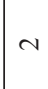 & $\sim$ & $\begin{array}{l}\infty \\
\infty \\
+\end{array}$ & & $N$ & $N$ & $\sim$ & - & - & $\begin{array}{l}\infty \\
\infty \\
i\end{array}$ & N & $\stackrel{t}{0}$ & & $N$ & & $N$ \\
\hline & 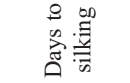 & & ర్ర & $\tilde{\sigma}$ & $\widetilde{్}$ & $\widetilde{్}$ & $\bar{\sigma}$ & 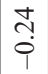 & & $\widehat{\sigma}$ & 6 & 6 & $\widetilde{\sigma}$ & $\vec{\sigma}$ & $\begin{array}{l}n \\
n \\
i \\
0\end{array}$ & ชิ & 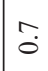 & & $\overline{6}$ & & $\overline{6}$ \\
\hline & 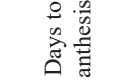 & & 8 & $\vec{\sigma}$ & 5 & 8 & 8 & $\frac{0}{9}$ & & $\vec{\sigma}$ & $\vec{\sigma}$ & $\bar{\sigma}$ & 8 & 8 & ले & $\bar{\sigma}$ & $\stackrel{0}{0}$ & & 8 & & 8 \\
\hline & 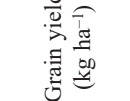 & & 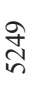 & $\frac{n}{\infty}$ & 竞 & $\stackrel{n}{\tilde{q}}$ & $\begin{array}{l}\hat{n} \\
\text { n. }\end{array}$ & $\begin{array}{l}\infty \\
\infty \\
0 \\
0\end{array}$ & & $\begin{array}{l}0 \\
\dot{4} \\
i n\end{array}$ & 茼 & के & $\begin{array}{l}0 \\
8 \\
0 \\
0\end{array}$ & $\begin{array}{l}\infty \\
\tilde{n}\end{array}$ & $\hat{o}$ & in & 导 & & $\begin{array}{l}\infty \\
\tilde{O} \\
i n\end{array}$ & & $\mid \begin{array}{l}\tilde{D} \\
\vec{b}\end{array}$ \\
\hline & 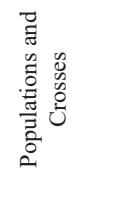 & 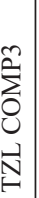 & 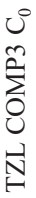 & 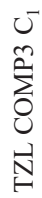 & $\begin{array}{l}\mathcal{N} \\
\tilde{N} \\
\hat{\beta} \\
0 \\
\mathcal{N} \\
\mathcal{N}\end{array}$ & 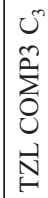 & \begin{tabular}{l}
$U^{+}$ \\
$\hat{N}$ \\
$\sum_{0}^{+}$ \\
$\mathcal{O}$ \\
\multirow{N}{N}{}
\end{tabular} & 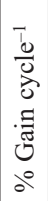 & $\begin{array}{l}\sum^{ \pm} \\
0 \\
\dot{U} \\
\vec{N}\end{array}$ & 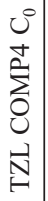 & $\begin{array}{l}U \\
\pm \\
\stackrel{\Delta}{\Delta} \\
0 \\
\cup \\
\vec{N}\end{array}$ & 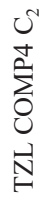 & $\begin{array}{l}U^{m} \\
+ \\
\sum_{0}^{+} \\
0 \\
\vec{N}\end{array}$ & $\begin{array}{l}U^{+} \\
\pm \\
\sum^{+} \\
0 \\
U^{\prime} \\
\vec{N}\end{array}$ & 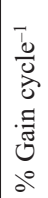 & 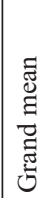 & 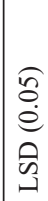 & 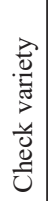 & 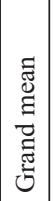 & 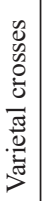 & 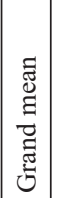 \\
\hline
\end{tabular}




\section{Response to selection in parental populations and varietal crosses}

This trial was conducted in eight test environments with diverse physical properties and growing conditions that resulted in average grain yields varying from 4,375 to $6,056 \mathrm{~kg}$ $\mathrm{ha}^{-1}$ for parental populations (Table 1). The changes in grain yield and most other agronomic traits from $\mathrm{C}_{0}$ to $\mathrm{C}_{4}$ did not follow any consistent trend for each composite. Days to silking, plant height, plant aspect and maize streak virus disease showed the highest improvement at $\mathrm{C}_{4}$ for TZL COMP3 whereas, grain yield, days to silking, husk cover and ears per plant showed the highest improvement at $\mathrm{C}_{4}$ for TZL COMP4 (Table 1). A negative percent gain per cycle for grain yield and a positive percent gain per cycle for ears per plant were obtained in TZL COMP3, whereas a positive percent gain per cycle were obtained for both grain yield and ears per plant in TZL COMP4. Linear regression coefficients of the parental populations over selection cycles were significant $(P<0.05)$ only for plant height, ear aspect, ears per plant and southern corn leaf rust. Three out of ten of the various selection cycles of the two composites had higher grain yields and were significantly $(P<0.05)$ different from the popular improved reference variety having a mean yield of $5,028 \mathrm{~kg} \mathrm{ha}^{-1}$ (Table 1).

Mean grain yields of varietal crosses varied from 5,616 to $6,545 \mathrm{~kg} \mathrm{ha}^{-1}$ (Table 2). Grain yield increased by $16.5 \%$ from $\mathrm{C}_{0} \times \mathrm{C}_{0}$ to $\mathrm{C}_{4} \times \mathrm{C}_{4}$. Relative to $\mathrm{C}_{0} \times \mathrm{C}_{0}$, ears per plant increased, whereas, flowering traits, plant height, husk cover, ear aspect, plant aspect and foliar diseases scores decreased after four selection cycles (Table 2). Linear regression coefficients of varietal crosses were significant $(P<0.05)$ for plant height, ear aspect and southern corn leaf rust scores but not for other traits. RRS was effective in attaining a genetic gain of $3.1 \%$ cycle $^{-1}$ for grain yield and $0.5 \%$ cycle $^{-1}$ for ears per plant. These improvements were associated with a reduction of 1.2 to $4.8 \%$ cycle $^{-1}$ in anthesis-silking interval, husk cover, ear aspect, plant aspect, maize streak virus disease, southern corn leaf rust and southern corn leaf blight (Table 2). The linear model for grain yield accounted for $69 \%$ of the total variation among cycles of selection.

\section{Changes in mid-parent heterosis (MPH)}

The varietal crosses showed positive MPH for grain yield and ears per plant. MPH for grain yield increased from $4 \%$ in $\mathrm{C}_{0} \times \mathrm{C}_{0}$ to $24 \%$ in $\mathrm{C}_{4} \times \mathrm{C}_{4}$, with an average $\mathrm{MPH}$ of $16 \%$. In contrast, $\mathrm{MPH}$ for ears per plant decreased from $4 \%$ in $\mathrm{C}_{0} \times \mathrm{C}_{0}$ to $2 \%$ in $\mathrm{C}_{4} \times \mathrm{C}_{4}$ (Table 3). MPH was negative and varied widely from $-0.8 \%$ in $\mathrm{C}_{0} \times \mathrm{C}_{0}$ to $-25.5 \%$ in $\mathrm{C}_{4} \times \mathrm{C}_{4}$ for other agronomic traits. Nonetheless, only MPH for plant aspect changed consistently from $-0.4 \%$ in to $-3.8 \%$ in $\mathrm{C}_{4} \times \mathrm{C}_{4}$. The improved varietal crosses $\left(\mathrm{C}_{1} \times \mathrm{C}_{1}\right.$, $\mathrm{C}_{3} \times \mathrm{C}_{3}$ and $\mathrm{C}_{4} \times \mathrm{C}_{4}$ ) produced significantly higher grain yield than their mid-parent. Genetic gains in heterosis were evident for grain yield, anthesis-silking interval, ear and plant aspects and southern corn leaf blight, but the linear responses for all measured traits were not significant. The changes in heterosis for grain yield and other agronomic traits were described by $\mathrm{R}^{2}$ values varying from 1 to $59 \%$ (Table 3 ). 


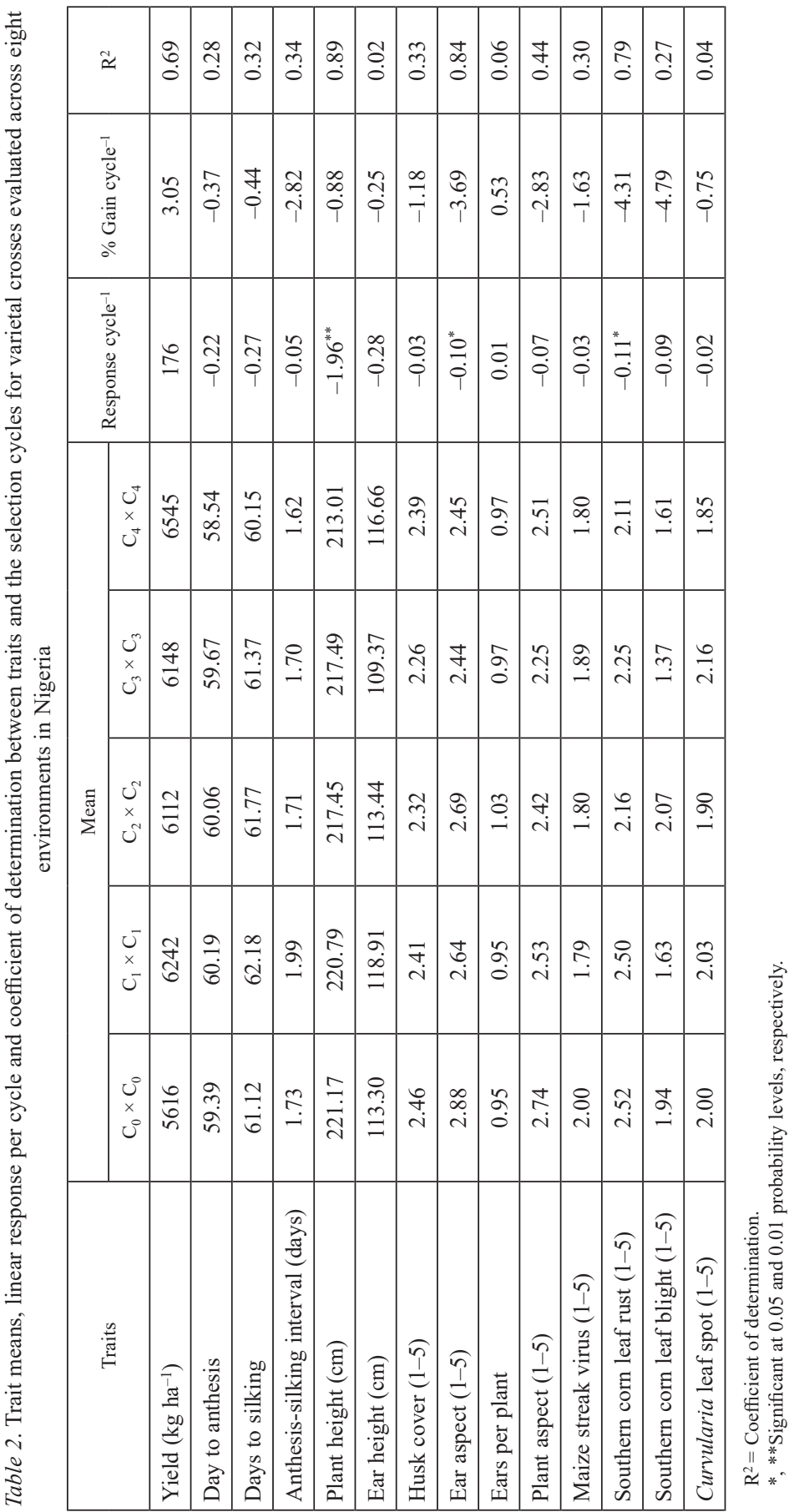




\begin{tabular}{|c|c|c|c|c|c|c|c|c|c|}
\hline & 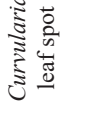 & $\underset{i}{\stackrel{\sim}{i}}$ & $\stackrel{\text { f }}{\rightarrow}$ & $\hat{\widehat{i}}$ & $\underset{\vec{\sigma}}{\sim}$ & $\stackrel{8}{i}$ & $\begin{array}{l}\text { J़ } \\
\stackrel{9}{9}\end{array}$ & $\stackrel{m}{?}$ & $\overrightarrow{0}$ \\
\hline \multirow{13}{*}{$\sum_{0}^{\frac{T}{0}}$} & 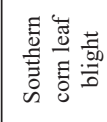 & $\begin{array}{l}\text { Oo } \\
\dot{i}\end{array}$ & $\stackrel{\infty}{\stackrel{\infty}{\varphi}}$ & \begin{tabular}{l}
$\infty$ \\
$\stackrel{0}{0}$ \\
\hdashline
\end{tabular} & $\begin{array}{l}\text { * } \\
\infty \\
n \\
n \\
i\end{array}$ & $\begin{array}{l}\vec{m} \\
\text { †े }\end{array}$ & $\frac{n}{i}$ & $\stackrel{\overrightarrow{0}}{i}$ & $\underset{\Xi}{*}$ \\
\hline & 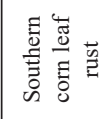 & $\begin{array}{l}\mathbb{J} \\
\stackrel{i}{i}\end{array}$ & $\stackrel{n}{\uparrow}$ & $\stackrel{+}{\stackrel{5}{1}}$ & $\stackrel{\infty}{\stackrel{\infty}{-}}$ & $\frac{0}{6}$ & $\begin{array}{l}\stackrel{0}{\sim} \\
\stackrel{+}{+}\end{array}$ & 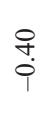 & $\stackrel{\overline{0}}{\circ}$ \\
\hline & 㥑 & $\begin{array}{l}\varkappa \\
\tilde{n}\end{array}$ & $\begin{array}{l}\stackrel{0}{0} \\
\frac{0}{1}\end{array}$ & $\underset{\mathfrak{o}}{\stackrel{0}{1}}$ & $\stackrel{\circ}{\circ}$ & ते & $\begin{array}{l}\infty \\
\infty \\
\dot{p}\end{array}$ & $\stackrel{\infty}{=}$ & $\stackrel{8}{\circ}$ \\
\hline & 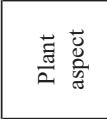 & $\stackrel{\infty}{\dddot{\infty}}$ & $\hat{i ̂}$ & ثֶ & 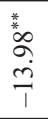 & $\stackrel{\hat{p}}{i}$ & nू. & $\stackrel{n}{n}$ & $\stackrel{\text { İ }}{0}$ \\
\hline & 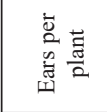 & $\stackrel{\text { f }}{+}$ & $\underset{\text { dै }}{\text { ì }}$ & $\vec{n}$ & $\begin{array}{l}\stackrel{\infty}{\infty} \\
\dot{+}\end{array}$ & $\vec{\sim}$ & $\stackrel{?}{\stackrel{f}{*}}$ & $\stackrel{ \pm}{9}$ & $\stackrel{\overrightarrow{0}}{\circ}$ \\
\hline & 穿 & $\stackrel{N}{0}$ & $\stackrel{\hat{a}}{\stackrel{0}{1}}$ & $\underset{0}{\stackrel{0}{p}}$ & $\stackrel{m}{\underset{T}{T}}$ & 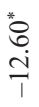 & $\begin{array}{c}\sigma \\
\text { ô } \\
\infty\end{array}$ & $\underset{i}{\stackrel{\infty}{i}}$ & ڤ̂? \\
\hline & $\begin{array}{l}\text { 窇离 } \\
\text { 总 }\end{array}$ & $\stackrel{\text { ọ }}{.}$ & 吕 & ڤે & $\begin{array}{l}* \\
\infty \\
\infty \\
\infty\end{array}$ & 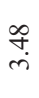 & $\stackrel{0}{\stackrel{1}{9}}$ & $\stackrel{\hat{\theta}}{i}$ & $\stackrel{\circ}{\circ}$ \\
\hline & 吉 & $\stackrel{1}{\infty}$ & $\vec{r}$ & $\stackrel{*}{\stackrel{*}{\sim}} \stackrel{+}{+}$ & $\frac{0}{0}$ & ñ? & $\bar{n}$ & $=$ & $\stackrel{\overrightarrow{0}}{\circ}$ \\
\hline & 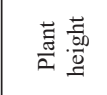 & $\stackrel{m}{\rightarrow}$ & $\begin{array}{l}\hat{b} \\
\dot{9}\end{array}$ & گू. & $\stackrel{\text { ָิ }}{-}$ & کे & $\frac{0}{0}$ & $\grave{\jmath}$ & $\frac{ \pm}{0}$ \\
\hline & 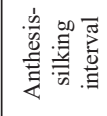 & $\begin{array}{l}\stackrel{+}{+} \\
\dot{+}\end{array}$ & $\begin{array}{l} \pm \\
\infty \\
\ddot{\lambda}\end{array}$ & $\begin{array}{c}n \\
n \\
\infty \\
1\end{array}$ & $\begin{array}{l}\stackrel{\infty}{N} \\
\stackrel{n}{n}\end{array}$ & $\begin{array}{l}\text { f } \\
\text { å }\end{array}$ & $\frac{\infty}{\dot{0}}$ & $\stackrel{+}{m}$ & $\stackrel{\circ}{\stackrel{0}{0}}$ \\
\hline & 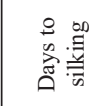 & $\stackrel{?}{i}$ & $\begin{array}{l}n \\
\infty \\
\dot{\varphi}\end{array}$ & $\stackrel{n}{1}$ & $\begin{array}{l}n \\
n \\
\varphi\end{array}$ & $\underset{i}{\stackrel{a}{i}}$ & in & $\stackrel{n}{0}$ & $\ddot{0}$ \\
\hline & 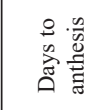 & 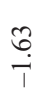 & $\stackrel{n}{i}$ & $\hat{n}$ & ஷे & 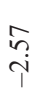 & ñ & $\stackrel{m}{9}$ & $\overrightarrow{0}$ \\
\hline & 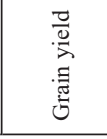 & $\underset{+}{\stackrel{+}{+}}$ & 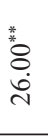 & $\begin{array}{l}\infty \\
\stackrel{0}{0} \\
0\end{array}$ & $\begin{array}{l}\stackrel{*}{\circ} \\
\stackrel{\infty}{\Xi} \\
\stackrel{-}{=}\end{array}$ & 离 & f̊ & $\stackrel{m}{m}$ & ָे \\
\hline & 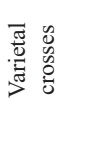 & $\begin{array}{l}u^{0} \\
\times \\
0\end{array}$ & $\begin{array}{l}u^{j} \\
x \\
u^{-}\end{array}$ & $\begin{array}{c}v^{N} \\
u_{2}\end{array}$ & $\begin{array}{l}m \\
\times \\
m\end{array}$ & $\begin{array}{l}U^{+} \\
U^{+}\end{array}$ & $\stackrel{\overparen{E}}{\Sigma}^{\mathbb{E}}$ & 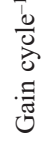 & $\approx$ \\
\hline
\end{tabular}




\section{Discussion}

In this study, significant environmental effect was observed for all traits measured, except for southern corn leaf blight and southern corn leaf rust diseases, which reveals variations in climatic and edaphic factors during evaluations of our trial. The significantly different means squares for grain yield and other agronomic traits observed after partitioning of the genotypes into parental population and varietal crosses indicate that genotypic variability exists in the two tropical maize composites and genetic gains may be achieved from continuous improvement of the composites. The observed interaction between genotypes and environments for grain yield, husk cover and ear aspect were caused mainly by the large climatic difference among test environments.

Responses to selection in the various selection cycles of the two composites in the present study were inconsistent for most traits while others remained unchanged until $\mathrm{C}_{4}$, which agrees with the reports of Souza Jr (1999) and Romay et al. (2011). Grain yield improved in TZL COMP4 at a rate of $0.9 \%$ per cycle, although the largest mean performance was reached during the third selection cycle, whereas, the improvement for grain yield was not consistent in TZL COMP3 resulting in a negative response, consistent with the negative, positive or lack of response observed in one or two populations improved through RRS in other breeding programs (Butruille et al. 2004; Moreno-Perez et al. 2004; Peña-Asin et al. 2013; Reis et al. 2013). A plausible explanation for negative response of TZL COMP3 could be because it was formed by crossing an orange maize variety from Thailand (Suwan 1-SR) with an adapted variety (TZB-SR) developed at IITA (Menkir et al. 2015). As the objective of the long-term RRS program was to develop a pair of source populations for white maize inbred lines with superior combining ability, only white lines with desirable agronomic traits were selected for testcross evaluation and recombination beginning with the formation of $\mathrm{C}_{2}$ in TZL COMP3. The selection against ears with yellow to orange kernel color may have led to elimination of an array of alleles, contributing to the observed inconsistent changes in grain yield from cycle to cycle.

The response in the varietal crosses for grain yield and other agronomic traits was greater than the response of the various selection cycles of the two composites, suggesting that dominance was the major type of gene action involved (Keeratinijakal and Lamkey 1993; Souza Jr and Pinto 2000). Inconsistent trends in grain yield observed in varietal crosses in the present study was consistent with the results reported in other studies (Romay et al. 2011; Peña-Asin et al. 2013). The linear response obtained for grain yield in our program was similar to the response obtained in other RRS programs (Coors 1999; Peña-Asin et al. 2013). This increase in grain yield was accompanied by improvements in number of ears per plant, shorter ASI, desirable plant height, husk cover, plant and ear characteristics and better resistance to foliar diseases. Increase in number of ears per plant observed in this present study was desirable as this trait is desirable for increased grain yield (Martin and Hallauer 1980). Similarly, the observed reduction in ASI in the present study could be described as sign of increased partitioning of assimilates to ears around flowering time (Edmeades et al. 1993). The changes in several traits of varietal crosses suggest that our selection procedure was effective in changing the frequencies of alleles with dominance effects (Falconer and Mackay 1996; Hallauer 1999). 
The observed increase in MPH for grain yield in our study was comparable to the findings reported in other studies (Souza Jr and Pinto 2000; Santos et al. 2007). MPH for grain yield was lowest at $\mathrm{C}_{0}$ and highest at $\mathrm{C}_{1}$ after which there was a consistent increase from $\mathrm{C}_{2}$ to $\mathrm{C}_{4}$, consistent with other studies (Eyherabide and Hallauer 1991; Ochieng and Kamidi 1992; Peña-Asin et al. 2013). The significant and desirable levels of heterosis over mid-parents obtained in the advanced selection cycle varietal crosses for grain yield, husk cover, ear and plant aspects and southern corn leaf bight, indicate that those varietal crosses have great potential as sources of elite and divergent inbred lines that can maximize heterosis in hybrids. Furthermore, positive mean MPH for grain yield (16\%) in the present study is comparable to the results obtained by Hallauer and Miranda (1995) and Carena (2005). Hence, the various selection cycles of the two composites were characterized by complementary improvement in favorable allelic frequencies and genetic divergence at loci with dominance effects which enhanced heterosis for grain yield and other agronomic traits, consistent with results in other studies (Falconer and Mackay 1996; Reis et al. 2014; Kolawole et al. 2017).

In conclusion, Our RRS program effectively improved the mean performances of the various selection cycles, genetic gain in heterosis for grain yield, resistance to foliar diseases and other agronomic traits of the varietal crosses. Once productive lines from advanced selection cycles of varietal crosses are identified they can become alternative profitable sources of seed. The advanced selection cycles of the two composites could be certified for seed quality and marketed in areas with limited market access.

\section{Acknowledgements}

This report is a part of $\mathrm{PhD}$ thesis research funded by the Alliance for a Green Revolution in Africa (AGRA) at West Africa Centre for Crop Improvement (WACCI), University of Ghana, Legon, and the International Institute of Tropical Agriculture (IITA). All the staff members of the Maize Improvement Unit at the International Institute of Tropical Agriculture (IITA), Ibadan, Nigeria, are appreciated for providing technical supports during planting, management, data collection, harvesting and threshing of the field trials conducted in multiple locations.

\section{References}

Betrán, F.J., Hallauer, A.R. 1996. Hybrid improvement after reciprocal recurrent selection in BSSS and BSCB1 maize populations. Maydica 41:360-367.

Butruille, D.V., Silva, H.D., Kaeppler, S.M., Coors, J.G. 2004. Response to selection and genetic drift in three populations derived from the golden glow maize population. Crop Sci. 44:1527-1534.

Carena, M.J., Hallauer, A.R. 2001. Expression of heterosis in Leaming and Midland corn belt dent populations. J. Iowa Acad. Sci. 108:73-78.

Carena, M.J. 2005. Maize commercial hybrids compared to improved population hybrids for grain yield and agronomic performance. Euphytica 141:201-208.

Carena, M.J., Wicks, III, Z.W. 2006. Maize population hybrids: An exploitation of U.S. temperate public genetic diversity in reserve. Maydica 51:201-208. 
Comstock, R.E., Robinson, H.F., Harvey, P.H. 1949. A breeding procedure designed to make maximum use of both general and specific combining ability. Agron. J. 41:360-367.

Coors, J.G. 1999. Selection methodologies and heterosis. In: Coors J.G., Pandey, S., Gerdes, J.T. (eds), Genetics and exploitation of heterosis in crops. ASA, CSSA, SSSA, Madison, Wisconsin. pp. 225-246.

Eberhart, S.A. 1964. Least squares method for comparing progress among recurrent selection methods. Crop Sci. 4:230-231.

Edmeades, G.O., Bolanos, J., Hernadez, M., Bello, S. 1993. Recent advances in breeding for drought tolerance in maize. In: Badu-Apraku, B., Akoroda, M.O., Ouedraogo, M., Quin, F.M. (eds), Contributing to food self-sufficiency: Maize research and development in West and Central Africa. Proc. Regional Maize Workshop, 29 May - 2 June 1995, IITA, Cotonou (Besini). pp. 404-420.

Eyherabide, G.H., Hallauer, A.R. 1991. Reciprocal full-sib recurrent selection in maize: I. Direct and indirect responses. Crop Sci. 31:952-959.

Falconer, D.S., Mackay, T.F.C. 1996. Introduction to quantitative genetics. 4th ed., Longman Group Ltd., Edinburgh, United Kingdom.

Hallauer, A.R., Miranda, J.B. 1995. Quantitative genetics in maize breeding, 2nd ed. lowa State University Press, Ames, IA, USA.

Hallauer, A.R. 1999. Temperate maize and heterosis. In: Coors, J.G., Pandey, S. (eds), Genetics and exploitation of heterosis in crops. ASA, CSSA, SSSA, Madison, Wisconsin. pp. 353-362.

Jenweerawat, S., Aekatasanawan, C., Laosuwan, P., Hallauer, A.R. 2010. Potential lines and hybrids developed from modified reciprocal recurrent selection in maize. Kasetsart J. (Nat. Sci.) 44:517-522.

Keeratinijakal, V., Lamkey, K.R. 1993. Responses to reciprocal recurrent selection in BSSS and BSCB1 maize populations. Crop Sci. 33:73-77.

Kolawole, A.O., Menkir, A., Gedil, M., Blay, E., Ofori, K., Kling, J.G. 2017. Genetic divergence in two tropical maize composites after four cycles of reciprocal recurrent selection. Plant Breeding 134:41-47.

Lamkey, K.R., Hallauer, A.R. 1987. Heritability estimated from recurrent selection experiments in maize. Maydica 32:61-78.

Martin, J.M., Hallauer, A.R. 1980. Seven cycles of reciprocal recurrent selection in BSSS and BSCB1 maize populations. Crop Sci. 20:599-603.

Menkir, A., Olowolafe, M.O., Ingelbrecht, I., Fawole, I., Badu-Apraku, B., Vroh, B.I. 2006. Assessment of testcross performance and genetic diversity of yellow endosperm maize lines derived from adapted $\times$ exotic backcrosses. Theor. Appl. Genet. 113:90-99.

Menkir, A., Kling, J.G., Anjorin, B., Ladejobi, F., Gedil, M. 2015. Evaluating testcross performance and genetic divergence of lines derived from reciprocal tropical maize composites. Maydica 60:M14.

Menz, M.A., Hallauer, A.R. 1997. Reciprocal recurrent selection of two tropical corn populations adapted to Iowa. Maydica 42:239-246.

Menz, M.A., Hallauer, A.R., Russell, W.A. 1999. Comparative response of two reciprocal recurrent selection methods in BS21 and BS22 maize populations. Crop Sci. 39:89-97.

MIP. 1996. Maize Improvement Program archival report, 1989-1992. Part I: Maize population improvement CID, IITA, Ibadan Nigeria.

Moreno-Perez, E.D., Lewis-Beck, D., Cervantes-Santana, T., Torres-Flores, J.I. 2004. Reciprocal recurrent selection in Mexican highland valleys maize populations under high and low soil nitrogen content. Agrociencia 38:305-311.

Mosisa, W., Wende, A., Berhanu, T., Legesse, W., Dagne, W., Girum, A. 2009. Performance of variety cross hybrids of Maize (Zea Mays L.) in the Mid-Altitude and Highland transition areas of Ethiopia. East African Journal of Sciences 3:80-86.

Ochieng, J.A.W., Kamidi, R.E. 1992. Response to eight cycles of reciprocal recurrent selection in Kitale synthetic II, Ecuador 573 and their variety cross. J. Genet. Breed. 46:315-320.

Olaniyan, A.B. 2015. Maize: Panacea for hunger in Nigeria. Afr. J. Plant Sci. 9:155-174.

Ordas, B., Butron, A., Alvarez, A., Revilla, P., Malvar, R.A. 2012. Comparison of two methods of reciprocal recurrent selection in maize (Zea mays L.). Theor. Appl. Genet. 124:1183-1191.

Paliwal, R.L. 2000. Hybrid maize breeding. In: Pliwal, R.L., Granados, G., Lafitte, H.R., Violic, A.D., Marathee, J.P. (eds.), Tropical maize improvement and production. FAO, Rome. pp. 143-160. 
Peña-Asin, J., Alvarez, A., Ordas, A., Ordas, B. 2013. Evaluation of three cycles of full-sib reciprocal recurrent selection in two maize populations from the Northeast of Spain. Euphytica 191:301-310.

Reis, M.C.D., Guedes, F.L., Abreu, G.B., de Souza, J.C. 2013. Reciprocal recurrent selection in maize enhances heterosis and ears yield. Euphytica 191:217-222.

Reis, M.C.D., Pádua, J.M.V., Abreu, G.B., Guedes, F.L., Balbi, R.V., de Souza, J.C. 2014. Estimates for genetic variance components in reciprocal recurrent selection in populations derived from maize singlecross hybrids. Sci. World J. Article ID 540152, http://dx.doi.org/10.1155/2014/540152.

Romay, M.C., Ordás, B., Revilla, P., Ordás, A. 2011. Three cycles of full-sib reciprocal recurrent selection in two Spanish maize populations. Crop Sci. 51:1016-1022.

Santos, M.F., Cậmara, T.M.M., Moro, G.V., Costa, E.F.N., De Souza Jr, C.L. 2007. Responses to selection and changes in combining ability after three cycles of a modified reciprocal recurrent selection in maize. Euphytica 157:185-194.

SAS Institute, 2010. Statistical Analysis Software (SAS). Users guide, SAS Inst. Inc. Cary, NC.

Singh, R.K., Chaudhary, B.D. 2006. Biometrical methods in quantitative genetic analysis. Kalyani Publishers, New Delhi, India.

Souza Jr, C.L. 1999. Recurrent selection and heterosis. In: Coors, J.G., Pandey, S., Gerdes, J.T. (eds), Genetics and exploitation of heterosis in crops. ASA, CSSA, SSSA, Madison, Wisconsin. pp. 247-255.

Souza Jr, C.L., Pinto, R.M.C. 2000. Responses to a short-term reciprocal recurrent selection procedure in maize. Maydica 45:21-28.

Souza Jr, C.L., Sanzio, C.L.B., Gustavo, V.M. 2010. Performance of maize single-crosses developed from populations improved by a modified reciprocal recurrent selection. Sci. Agr. 67:198-205.

\section{Electronic Supplementary Material (ESM)}

Electronic Supplementary Material (ESM) associated with this article can be found at the website of CRC at https://akademiai.com/loi/0806

Electronic Supplementary Table S1. Parental populations and varietal crosses of TZL COMP3 and TZL COMP4 along with a check variety used in the study

Electronic Supplementary Table S2. Mean squares from the combined analyses of variances for grain yield and other traits of the parent lines and varietal crosses evaluated across eight environments in Nigeria

Electronic Supplementary Table S3. Grain yield of the parental populations, varietal crosses and a check variety evaluated across eight environments in Nigeria 